\title{
La modernización de los comportamientos de fecundidad en España durante el siglo XX. Un estudio a nivel provincial para las generaciones nacidas en la primera mitad del siglo XX
}

\author{
Roser Nicolau \\ Daniel Devolder \\ Eva Panareda \\ Universitat Autònoma de Barcelona \\ Departament d'Economía i Història Econòmica i Centre d'Estudis Demogràfics \\ 08193 Bellaterra \\ rnicolau@ced.uab.es
}

Recibido: 10-10-2007

Aceptado: 05-05-2008

\section{Resumen}

En este trabajo, hacemos un análisis exploratorio de una fuente poco utilizada para el estudio de los comportamientos demográficos provinciales, la pregunta en los censos sobre el número de hijos nacidos vivos que tuvieron las mujeres antes del censo. Hacemos un análisis temporal de la progresión del control de la fecundidad y de coherencia territorial de las evoluciones, presentando pistas sobre las causas probables de los cambios detectados. El principal tipo de indicador utilizado es la probabilidad de agrandamiento para cada rango de nacimiento. Analizamos los valores de estos indicadores e introducimos un método de detección del control de la fecundidad a partir del patrón por rango de estas probabilidades.

Palabras clave: fecundidad, generación, rango de nacimiento, control de la fecundidad, probabilidad de agrandamiento, España, provincias, siglo XX.

Abstract. The Modernization of the Fertility Behaviours in Spain during the 20th Century. A study at the provincial level for the cohorts born during the first part of the Century

In this paper we undertake an exploratory analysis of regional fertility behaviours, using a data source still underutilized for that purpose: the results of the census question on the number of women's live births until that moment. We analyse the progression of fertility control across cohorts and the territorial coherency of the evolution in time. We discuss what might have been the causes of the observed changes. The main fertility indicator used is the cohort parity progression ratio for each birth order. We discuss the main patterns of values of these ratios found at the regional level and across cohorts. We also use an indirect method of detection of fertility control, based on the expected pattern of the parity ratios by order in a natural fertility population.

Key words: fertility, cohort, birth order, fertility control, parity progression ratio, Spain, region, twentieth century. 


\section{Sumari}

\begin{tabular}{|c|c|}
\hline $\begin{array}{r}\text { Introducción } \\
\text { 1. Validación de los datos }\end{array}$ & $\begin{array}{l}\text { 5. La progresión de la limitación } \\
\text { de la fecundidad }\end{array}$ \\
\hline $\begin{array}{l}\text { 2. Evolución de la fecundidad por rango } \\
\text { y de su dispersión provincial }\end{array}$ & $\begin{array}{l}\text { 6. Estudio del control de la fecundidad } \\
\text { a partir de la generación 1896-1900 }\end{array}$ \\
\hline 3. La infecundidad & Conclusiones \\
\hline 4. El modelo del hijo único & Referencias bibliográficas \\
\hline
\end{tabular}

\section{Introducción}

El estudio de la evolución histórica de la fecundidad española a nivel territorial, en las provincias o en las comunidades autónomas, esta sometido a dos condicionantes que han dificultado los progresos en los años recientes. Por un lado, hay lagunas importantes en la publicación de los datos oficiales de nacimientos por edad de los padres a nivel provincial y, por otro lado, los resultados del proyecto sobre la transición de fecundidad a nivel europeo, coordinado desde la Universidad de Princeton, tienden a apabullar a los estudiosos (Coale y Watkins, 1986).

En este trabajo, usamos una fuente alternativa para el estudio de la fecundidad en España, que puede servir para analizar las diferencias a nivel provincial y su evolución en el tiempo. Así pretendemos suplir, en parte, las carencias de las estadísticas oficiales. Estas estadísticas permiten reconstruir la fecundidad en distintos períodos y generaciones para el conjunto de España a lo largo del siglo XX (Saez, 1979). No obstante, el Instituto Nacional de Estadística no publicó los datos de nacimientos por edad de los padres a nivel provincial para el periodo 1920-1974, y muy pocos estudiosos han podido tener acceso a las tabulaciones manuscritas (Cabré, 1999). Esperemos que lleguen a publicarse estos datos, pero, incluso en este caso, el uso de estas tabulaciones tendría, desde nuestro punto de vista, una limitación importante, la de carecer del detalle de los rangos de nacimiento, por lo cual tiene sentido ver si, con esta fuente alternativa a los indicadores indirectos utilizados en el proyecto de Princeton, es posible estudiar con más detalle el proceso de modernización de los comportamientos de fecundidad durante el siglo. Presentaremos aquí los principales resultados obtenidos con esta fuente, pero sin entrar en un análisis detallado de las causas de las evoluciones, que todavía se tiene que desarrollar.

La fuente que aquí se ha utilizado es la tabulación de las respuestas a la pregunta sobre el número de hijos nacidos vivos que se introdujo en España en el censo de 1920, y que se repitió en los censos de 1930, 1970, 1981 y 1991. Concretamente, hemos utilizado en este trabajo los datos de los censos de 1970 y 1991. Descartamos la información de los censos de 1920 y 1930, porque, en la distribución del número de hijos según la edad de la madre, las mujeres de 45 años y más están agrupadas, lo que dificulta un estudio de las diferencias 
entre generaciones como el que pretendemos hacer. Tampoco utilizamos los datos del censo de 1981, porque los resultados se solapan totalmente con los datos de 1970 y 1991, y también por algunas dudas que teníamos sobre la coherencia interna de los datos de este censo.

Los indicadores de Princeton permiten estimar el nivel de la fecundidad total y legítima, así como el nivel de la nupcialidad, con un grado de aproximación muy aceptable. El uso principal de estos indicadores, en el marco del proyecto de Princeton, fue establecer el período a partir del cual empezó la difusión del control de la fecundidad, pero estos indicadores tienen limitaciones importantes para dicho fin: la principal es que miden la fecundidad en términos agregados, cuando el control de la fecundidad afecta más a ciertos rangos que a otros. Otra limitación es que no permiten detectar los efectos del uso de la contracepción sobre el nivel de la fecundidad de forma directa. Así, el criterio principal utilizado en el marco de este proyecto es el descenso de un $10 \%$ o más en el nivel del indicador de fecundidad legítima, a partir del año que corresponde al nivel más alto registrado desde el inicio de la serie. Esto obliga a disponer de una serie temporal con al menos dos observaciones en las que la fecundidad legítima sea estable y elevada, antes de que se detecte el descenso. Y como el criterio es indirecto, es imposible afirmar que la caída observada no fue precedida por un declive anterior al período en el que aparecen las fuentes estadísticas modernas. Esta limitación es especialmente problemática, porque, en muchos países, el declive de la fecundidad estaba en marcha o bien había empezado ya en el momento en el que se publicaron las estadísticas demográficas necesarias para el cálculo de los indicadores de Princeton.

Otra limitación significativa de estos indicadores es que utilizan un enfoque de período, cuando un enfoque por generación parece el más adecuado para detectar un proceso de difusión del control de la fecundidad. En efecto, los indicadores de Princeton reflejan el nivel medio de la fecundidad de todas las generaciones en edad fértil en cada momento y no permiten realizar un estudio separado de cada generación. Es una limitación importante, porque, en general, los procesos de difusión de comportamientos nuevos suelen afectar más a algunas generaciones que a otras y, en particular, las generaciones más jóvenes suelen ser las primeras en experimentar los cambios.

La ventaja principal de la fuente utilizada en este trabajo, las tabulaciones de las mujeres según el número de hijos nacidos vivos, es que hace posible calcular indicadores como las probabilidades de agrandamiento de las familias para cada rango, que permiten detectar de forma directa los efectos del uso de métodos de control de la fecundidad legítima. De hecho, el patrón por rango de la fecundidad es indicativo de la presencia de control, de la misma manera que el patrón por edad de la fecundidad legítima se puede utilizar para comprobar la existencia y medir la intensidad del control de la fecundidad legítima (Coale y Trussel, 1974; Ryder, 1986; David y Sanderson, 1990; Okun, 1994). Otro interés de estos datos es que permiten estudiar la fecundidad de las generaciones, lo que, como hemos señalado, es especialmente apropiado para la observación de la difusión de los comportamientos. 


\section{Validación de los datos}

Pero estos datos presentan también problemas, debido al hecho de que se trata de una información de tipo retrospectiva para las mujeres residentes en cada provincia en el momento del censo, y no una fuente de datos de tipo continua, que permita seguir la fecundidad en el momento y en el lugar en que se han producido los nacimientos. Otro inconveniente, al revés de lo que comentábamos para los indicadores de Princeton, es que, al tratarse de datos de tipo longitudinal, es más difícil establecer relaciones con cambios rápidos del entorno social, como los que se producen durante una guerra o una fase de crecimiento económico acelerado o de depresión. Otra clase de problema, esta vez relacionado con el uso que vamos a hacer de aquella fuente, es que, en las publicaciones de los censos, no se elaboraron tabulaciones por separado de las mujeres según la edad en que se casaron, lo que crea dificultades para la aplicación de métodos de detección del grado de control de la fecundidad. Esta deficiencia se puede suplir en parte con el uso de la encuesta sociodemográfica del año 1991, pero con el inconveniente de que es más difícil trabajar a un nivel provincial con esta fuente alternativa, debido a los problemas de representatividad de los resultados obtenidos a esta escala territorial.

La utilización de las tabulaciones de hijos nacidos vivos para un estudio de la fecundidad a nivel provincial plantea problemas y dudas acerca de los resultados. En efecto, tratándose de una fuente de tipo retrospectiva, podrían estar afectados por distintos sesgos:

- Errores en los datos, porque una parte de las mujeres no contestaron o indicaron un número de hijos nacidos vivos incorrecto, generalmente debido a la omisión de los hijos muertos en los primeros meses.

- Efectos de selección por la mortalidad diferencial de las mujeres por estado civil o por número de partos.

- Efectos de selección por las migraciones, ya que podríamos tener migraciones de provincias con alta fecundidad a provincias con baja fecundidad o a la inversa, algo que no podemos corregir con los datos de que disponemos, ya que las tabulaciones son para las mujeres residentes en cada provincia en el momento del censo.

- Efectos aleatorios debidos a los efectivos reducidos de las poblaciones provinciales, teniendo en cuenta que, además, trabajamos con grupos de mujeres clasificadas por estado civil, edad y paridad.

Estos problemas o sesgos han sido estudiados tanto para los datos censales de España (Recaño y Luxan, 1997; Devolder, Nicolau y Panareda, 2004), como de otros países, en particular para los Estados Unidos (Morgan, 1991). La conclusión de estos estudios es que los efectos de los sesgos son controlables, sobre todo si se limita el análisis a las respuestas de las mujeres de menos de 70 años, si se aplican las correcciones necesarias en el caso de las mujeres sin hijos que figuran incorrectamente en las casillas de "No sabe y/o no contesta» y si se es especialmente cauto en el estudio de los cambios de la fecundidad en 
las regiones urbanas con una fuerte inmigración, es decir, en el caso español, fundamentalmente para las provincias de Barcelona y Madrid.

\section{Evolución de la fecundidad por rango y de su dispersión provincial}

El gráfico 1 relaciona la evolución de la fecundidad total de las generaciones con sus componentes por rango evaluados con las probabilidades de agrandamiento, que indican, entre las mujeres que tuvieron $n$ hijos, la proporción de ellas que tuvieron un hijo más. La fecundidad total está medida con la descendencia final, calculada aquí para todas las mujeres y para las mujeres casadas al menos una vez, es decir, no solteras (ns). La diferencia entre ambas es debida al nivel de la soltería y, concretamente, al hecho de que cerca del 10\% de las mujeres no se casaron nunca y, por regla general, no tuvieron hijos. Para la fecundidad por rango, el indicador para el primer hijo está también medido para el total de las mujeres $\left(a_{0}\right)$ y para las mujeres casadas al menos una vez $\left(a_{0} n s\right)$. De nuevo, la diferencia entre estas dos proporciones de mujeres que tuvieron al menos un hijo se explica en gran parte por la soltería, pero, para las generaciones más antiguas, la viudedad desempeñó también un papel significativo, tal como veremos más adelante. En cambio, no presentamos por separado los valores de los indicadores de los rangos superiores para el total de las mujeres y las mujeres no solteras, es decir, la proporción de mujeres con un hijo que tuvieron un segundo $\left(a_{1}\right)$ y las proporciones similares para las

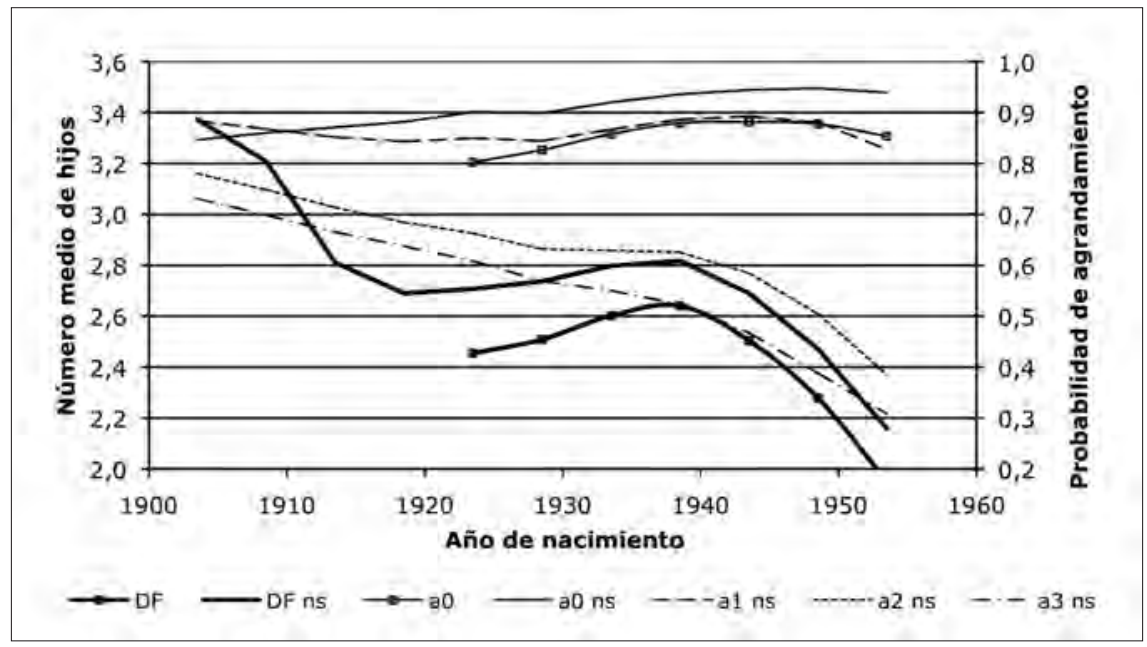

Gráfico 1. Evolución de la descendencia final y de las probabilidades de agrandamiento por generación para el conjunto de España.

Nota: DF: descendencia final; $a_{0}, a_{1}, a_{2}, a_{3}$ : probabilidades de agrandamiento sucesivas; ns: mujeres no solteras (casadas al menos una vez).

Elaboración propia de los datos de los censos de 1970 y 1991. 
mujeres con dos o tres hijos que tuvieron al menos un hijo más $\left(a_{2} \mathrm{y} a_{3}\right)$. La razón es que los valores de estas probabilidades son muy parecidos para los dos conjuntos de mujeres y por ello representamos solamente una curva.

Observamos, en el gráfico 1, que la evolución a largo plazo de las descendencias finales está explicada por la evolución de $a_{2}$ y $a_{3}$, es decir, las probabilidades asociadas con el tercer y el cuarto hijos. En cambio, las probabilidades de tener un primer hijo y un segundo hijo aumentaron de forma casi continua de generación en generación, y solo empezaron a disminuir recientemente, en las mujeres nacidas en la década de 1950 . Se observa también que la proporción de mujeres que tienen al menos un hijo aumenta desde las primeras generaciones y se acerca al máximo biológico en las generaciones nacidas en la década de 1940.

Esta evolución contrapuesta de la fecundidad por rango, con un aumento para los rangos inferiores y un descenso a partir del tercer hijo, confirma la validez de nuestras hipótesis iniciales, que nos llevaban al reemplazo de indicadores globales como los de Princeton para estudiar los procesos de cambio, en este caso, de control progresivo de la fecundidad.

Como introducción al estudio territorial, podemos examinar primero la dispersión provincial de estos indicadores (gráfico 2). En primer lugar, es remarcable la tendencia a la mayor homogeneidad para todas las probabilidades menos la segunda, que indica la proporción de mujeres que, teniendo un hijo, progresan hacia el segundo. Otra evolución destacable es el aumento de la dispersión para las probabilidades de rango superior entre las generaciones nacidas a finales del siglo XIX. Esta mayor dispersión se explica por un desfase cronológico entre provincias de la extensión del control de la fecundidad, que afectó sobre todo a estos rangos. Las familias de algunas provincias se adelantaron en la limitación de la fecundidad, lo que provocó un aumento de las diferencias a nivel territorial, que se redujeron luego, cuando se generalizaron estos comportamientos de limitación de la descendencia a un número mayor de familias en todo el territorio.

De forma general, lo que sugiere la evolución de los indicadores de intensidad de la fecundidad, así como los de su dispersión, es la progresiva marcha hacia una mayor homogeneidad de los comportamientos, tanto entre las mujeres como a nivel territorial. En primer lugar, podemos hablar de una mayor igualdad entre mujeres, porque la primera maternidad (o $\left.a_{0}\right)$ es muy elevada en las generaciones más recientes y, además, hay una marcada reducción de las diferencias entre las provincias. En segundo lugar, observamos, tanto a nivel nacional como provincial, una reducción de las diferencias en el tamaño de las familias. Debido al descenso rápido de las probabilidades para los rangos superiores a tres, se reduce el peso de las familias de tres o más hijos y las familias numerosas desaparecen progresivamente, en beneficio de las familias de uno y dos hijos. Pero este mecanismo de mimetismo o de normalización de los comportamientos encuentra una excepción notable en la proporción de madres que tuvieron al menos un segundo hijo, puesto que las diferencias a nivel territorial, que eran ya notables para las generaciones nacidas a finales 


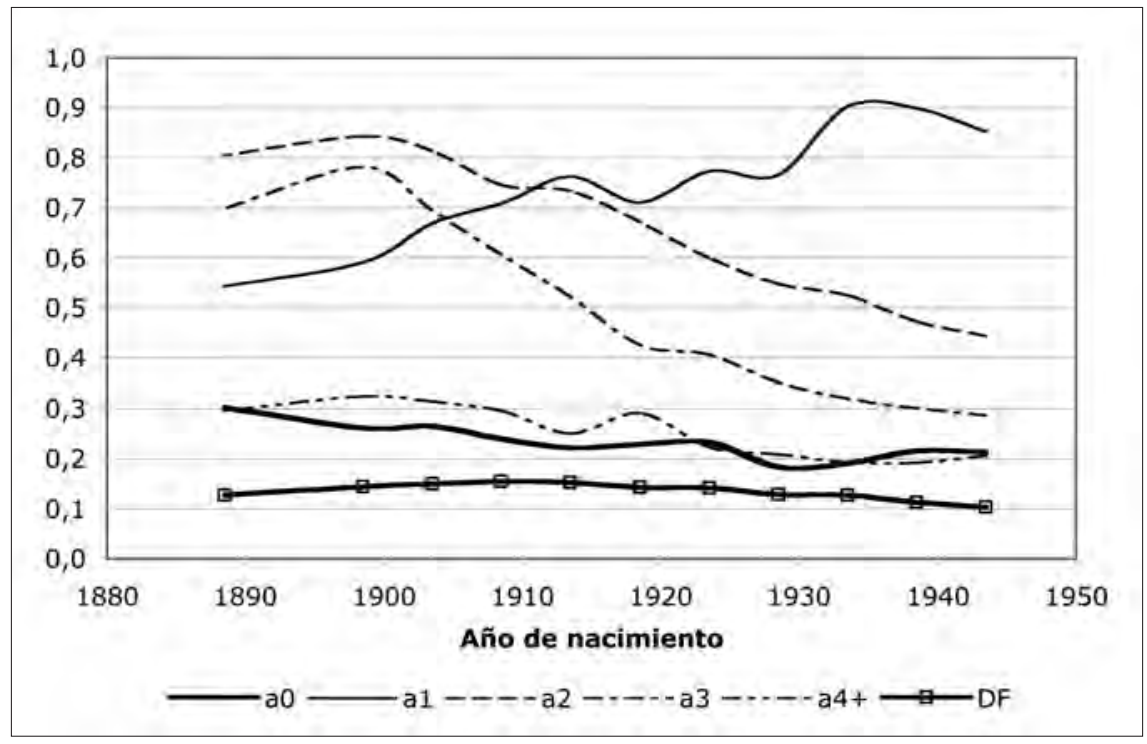

Gráfico 2. Dispersión de la fecundidad total y por rango. Coeficientes de variación provincial de la descendencia final y de las probabilidades de agrandamiento.

Nota: todos los valores son para las mujeres casadas al menos una vez. Los coeficientes de variación, es decir, la desviación estándar dividida por la media, están calculados a partir del logit de las probabilidades. Los valores de los indicadores de fecundidad provinciales están obtenidos a partir de una explotación propia de los datos de los censos de 1970 y 1991.

del siglo XIX, se ampliaron de forma considerable durante el siglo XX. Como lo veremos más adelante, esto se explica por la presencia de un grupo de provincias en las que el modelo de familia de hijo único atraía a un sector amplio de la población.

\section{La infecundidad}

\section{a. Evolución a nivel nacional}

Las series del gráfico 3 muestran la evolución de la infecundidad de las generaciones españolas nacidas a finales del siglo XIX hasta las generaciones nacidas en la década de 1950. El indicador utilizado es la proporción de mujeres que no tienen hijos al final de su vida fértil. Esta proporción es el complemento a uno de la proporción de mujeres que tuvieron al menos un hijo antes del final de su vida fértil, en torno a los cincuenta años (el indicador $a_{0}$ ). Observamos que la infecundidad era elevada en las generaciones nacidas a finales del siglo XIX y principios del siglo XX. Entre un 25 y un 30\% de estas mujeres no tuvieron ningún hijo, lo que contrasta singularmente con la situación de las mujeres nacidas durante los años 1940, para las cuales esta proporción se redujo 


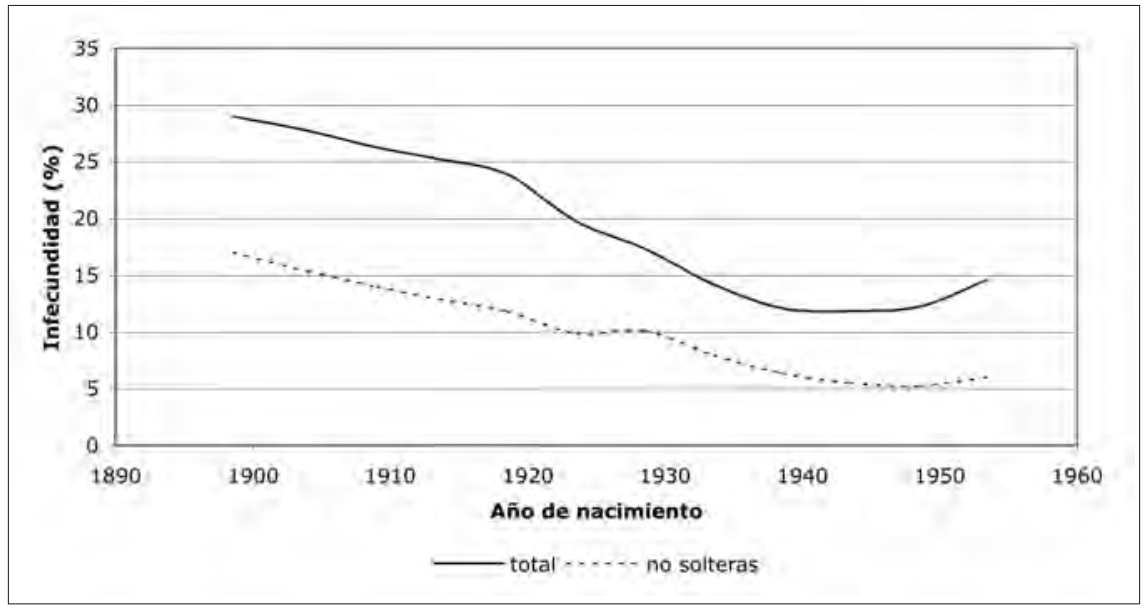

Gráfico 3. La evolución de la infecundidad por generaciones, para el conjunto de las mujeres y para las mujeres casadas alguna vez.

Fuente: cálculos directos a partir de los censos de 1970 y 1991. Los valores de infecundidad total para las generaciones nacidas antes de 1920 están estimados a partir de la infecundidad para las no solteras multiplicada por uno, menos la proporción de solteras de las generaciones correspondientes en los censos.

hasta un $12 \%$. Esta infecundidad elevada entre las mujeres que tuvieron a sus hijos en el periodo de entreguerras puede sorprender en un principio, pero esto no fue un fenómeno exclusivo de las mujeres españolas, puesto que los niveles de infecundidad en Estados Unidos y en Australia eran para aquellas generaciones similares, y fueron en general muy elevados en todos los países occidentales, como lo muestran, por ejemplo, Morgan (1991), Rowland (1998) y Devolder y Merino (2004).

La explicación de la elevada infecundidad remite, en el caso español, a la baja nupcialidad y a la elevada mortalidad adulta. Estos factores se pueden apreciar gracias a los datos del gráfico 4 . La proporción de solteras a los cincuenta años alcanzaba un $15 \%$ en las generaciones nacidas a principios del siglo XX. Como la fecundidad de esta mujeres era muy reducida (sus nacimientos representaban menos del 1\% del total de los nacimientos de la generación), la soltería llevaba a una reducción en la misma proporción del nivel de la fecundidad. Otro factor a tener en cuenta, esta vez para la infecundidad de las mujeres casadas alguna vez, es la mortalidad del cónyuge. La proporción de mujeres que permanecen casadas a los 45 años, es decir, con el marido en vida en aquella edad, alcanzaba el $72 \%$ del total en las generaciones nacidas a principios del siglo XX. Esto significa que, por diferencia y una vez restada la soltería, en torno al $15 \%$ de las mujeres de estas generaciones eran viudas o estaban separadas de su marido al final de su vida fértil. Esta proporción se redujo al 5\% para las mujeres nacidas hacia 1920. La proporción de viudas al 


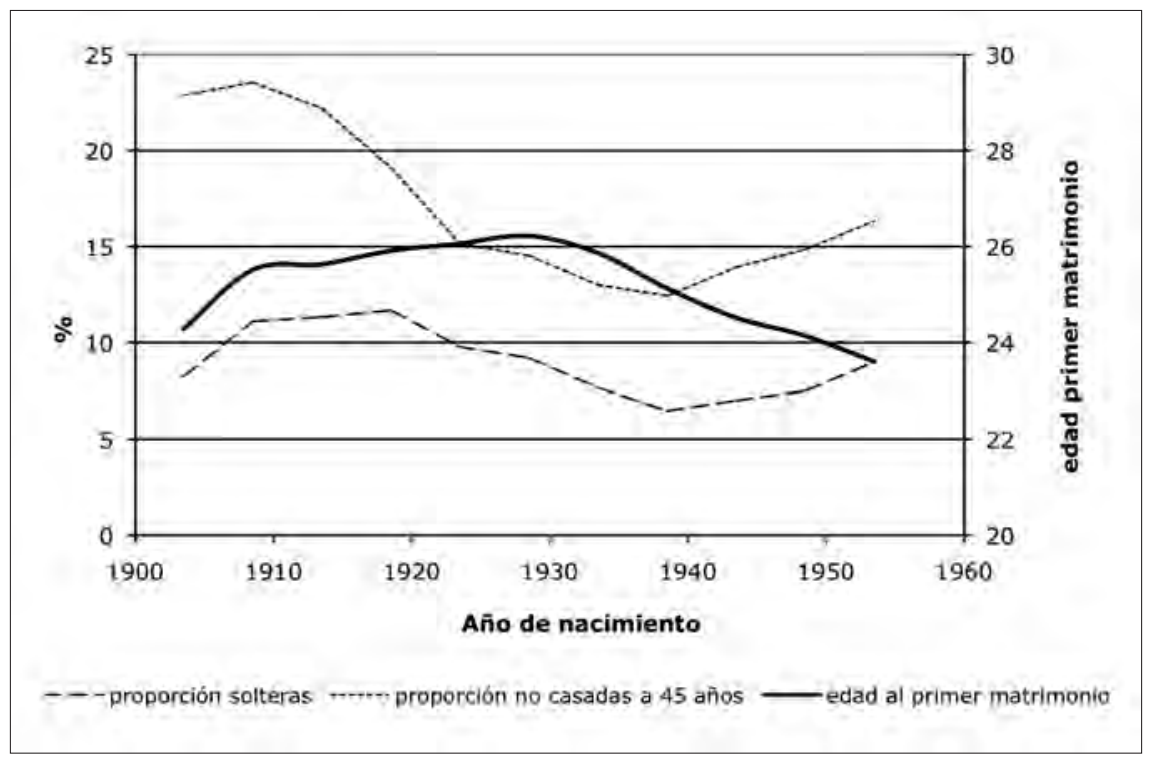

Gráfico 4. Evolución de la soltería, de la edad en el momento de contraer el primer matrimonio y del complemento a 100 de la proporción de casadas a los 45 años, por generación.

Fuente: Cabré 1999 para la soltería y la edad en el momento de contraer el primer matrimonio. La proporción de mujeres no casadas a los 45 años, esta obtenida a partir de una explotación propia de los datos de la Encuesta sociodemográfica de 1991, con una corrección para tener en cuenta la subestimación de la soltería en esta encuesta.

final de la vida fértil no influye de forma tan importante en la proporción de infecundidad como es el caso de la soltería, puesto que, para que así sea, la defunción del marido se tendría que producir antes de la primera concepción, lo que no es el caso en muchas ocasiones. Así, una proporción de viudas del $15 \%$ explicaría a lo sumo 2 o 3 puntos del $15 \%$ de infecundidad de las mujeres no solteras nacidas a finales del siglo XIX. Nuestro objetivo sería explicar casi 10 puntos de diferencia en la infecundidad de aquellas generaciones y las nacidas a finales de los años 1940 (ver gráfico 4).

Además de la soltería y la viudedad, tendríamos que considerar, por lo tanto, otros factores que intervinieron en la infecundidad. Uno de ellos pudo ser el aumento progresivo de la edad media en el primer matrimonio entre las generaciones de principios de siglo hasta 1920. No obstante, la suma de los factores estrictamente demográficos relacionados con la nupcialidad comentados hasta ahora no basta para explicar la infecundidad total de las mujeres casadas alguna vez. No podemos excluir, por lo tanto, el posible papel de factores como la salud para explicar esta infecundidad elevada, asociados, por ejemplo, con las enfermedades de transmisión sexual, aunque no tenemos ninguna información que nos permita afirmar esto con seguridad. 

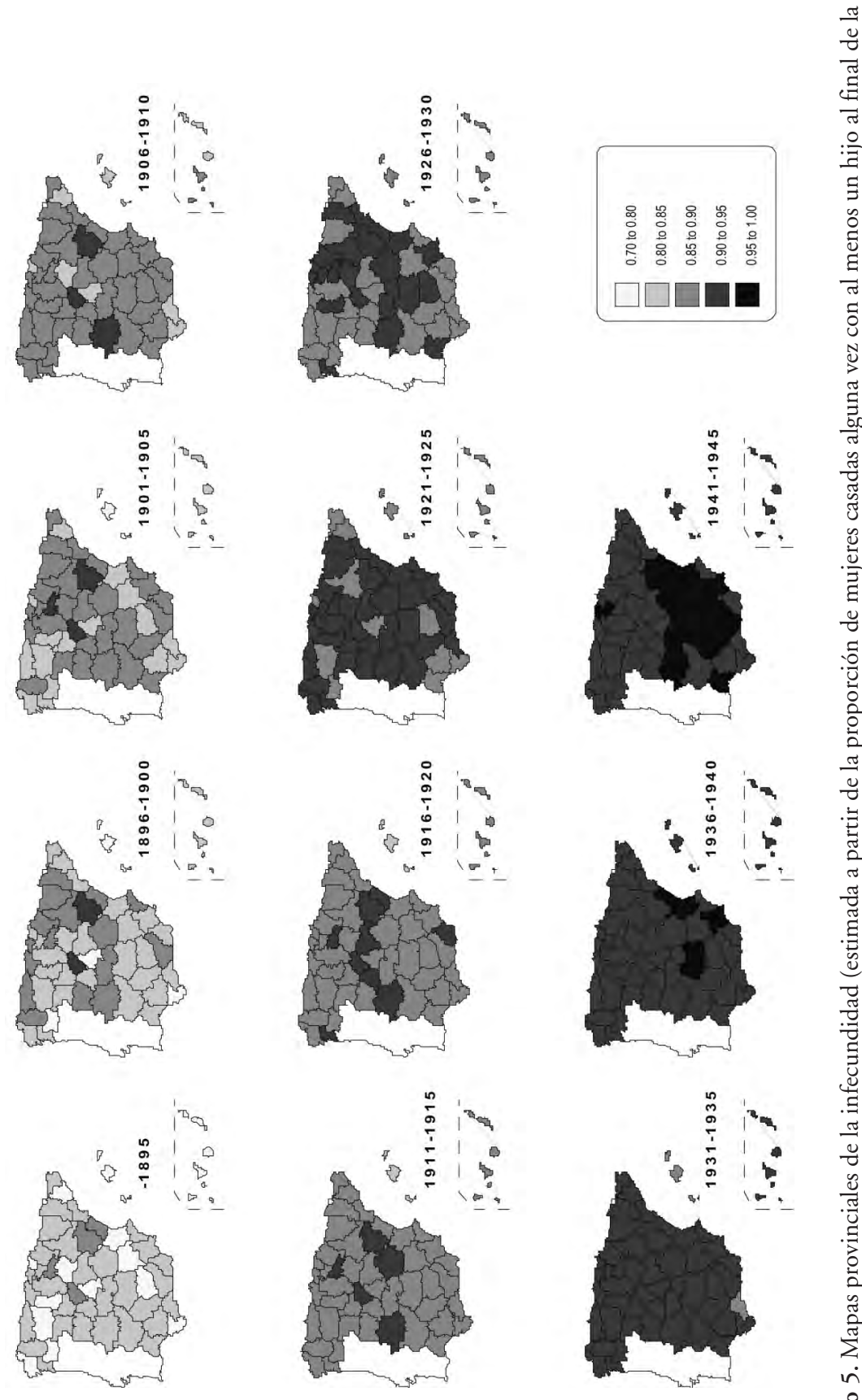


\section{b. Evolución a nivel provincial}

El descenso de la infecundidad a nivel nacional, acompañado de la reducción de la dispersión provincial en los valores de este indicador, tienen un claro reflejo en los datos del conjunto de mapas del gráfico 5. Estos mapas reproducen los valores del indicador $a_{0}$ para las mujeres no solteras, es decir, comparables con el complemento a uno de los valores de la curva inferior del gráfico 4. Para las generaciones más jóvenes examinadas, los mapas son casi de un solo color, con valores elevados de dichas proporciones, mientras que, para las generaciones nacidas a finales del siglo XIX, las diferencias son extremas, con zonas de alta infecundidad, superior al 20\%, que alternan con zonas de más baja infecundidad en el centro y el extremo norte, donde los valores se acercan más al 10\% (por ejemplo, para la generación 1896-1900, había diferencias del nivel de infecundidad del simple al doble entre provincias como Cádiz y Ourense y provincias de baja infecundidad como Teruel y Segovia). Estos valores altos de infecundidad y las diferencias territoriales para las generaciones más antiguas seguramente se podrían explicar en parte por los mismos factores comentados a nivel nacional, es decir, la importancia de la viudedad y el retraso de la edad de acceso al matrimonio. Pero, de nuevo, sería conveniente explorar otras posibles razones, puesto que las diferencias territoriales de nupcialidad femenina y de mortalidad masculina adulta no eran tan extremas como para poder explicar estas diferencias de infecundidad entre provincias, y sobre todo porque los patrones territoriales de nupcialidad y de mortalidad no se parecen entre sí. En este sentido, y como lo sugerimos antes, una posible razón podría ser la existencia de diferencias en la salud reproductiva. En efecto, se ha podido observar en muchos países con un nivel de desarrollo como el español del siglo XIX, que los niveles de fecundidad aumentaron antes de producirse el descenso histórico asociado con la transición demográfica. Estos aumentos de la fecundidad no controlada o "natural» podrían corresponder a mejoras en el nivel sanitario y nutritivo de las mujeres, que permitieron una reducción de la mortalidad intrauterina y una reducción de los niveles de esterilidad asociados a las enfermedades de transmisión sexual, que, en algunos casos, fueron combatidas muy eficazmente a partir de la década de 1940 con el uso de antibióticos (Dyson y Murphy, 1985; Wrigley, Davies y otros, 1997).

\section{El modelo del hijo único}

El conjunto de mapas del gráfico 6 presenta los valores provinciales para las distintas generaciones de la probabilidad $a_{1}$ estimada para las mujeres no solteras. Esta probabilidad muestra la proporción entre aquellas mujeres con al menos un hijo que tuvieron un segundo. Conviene resaltar de entrada que, a diferencia de $a_{0}$, esta proporción no depende del valor de la soltería o de la viudedad, y que la probabilidad para las mujeres no solteras tiene, de manera general, un valor muy similar a la misma probabilidad calculada para el conjunto de las 

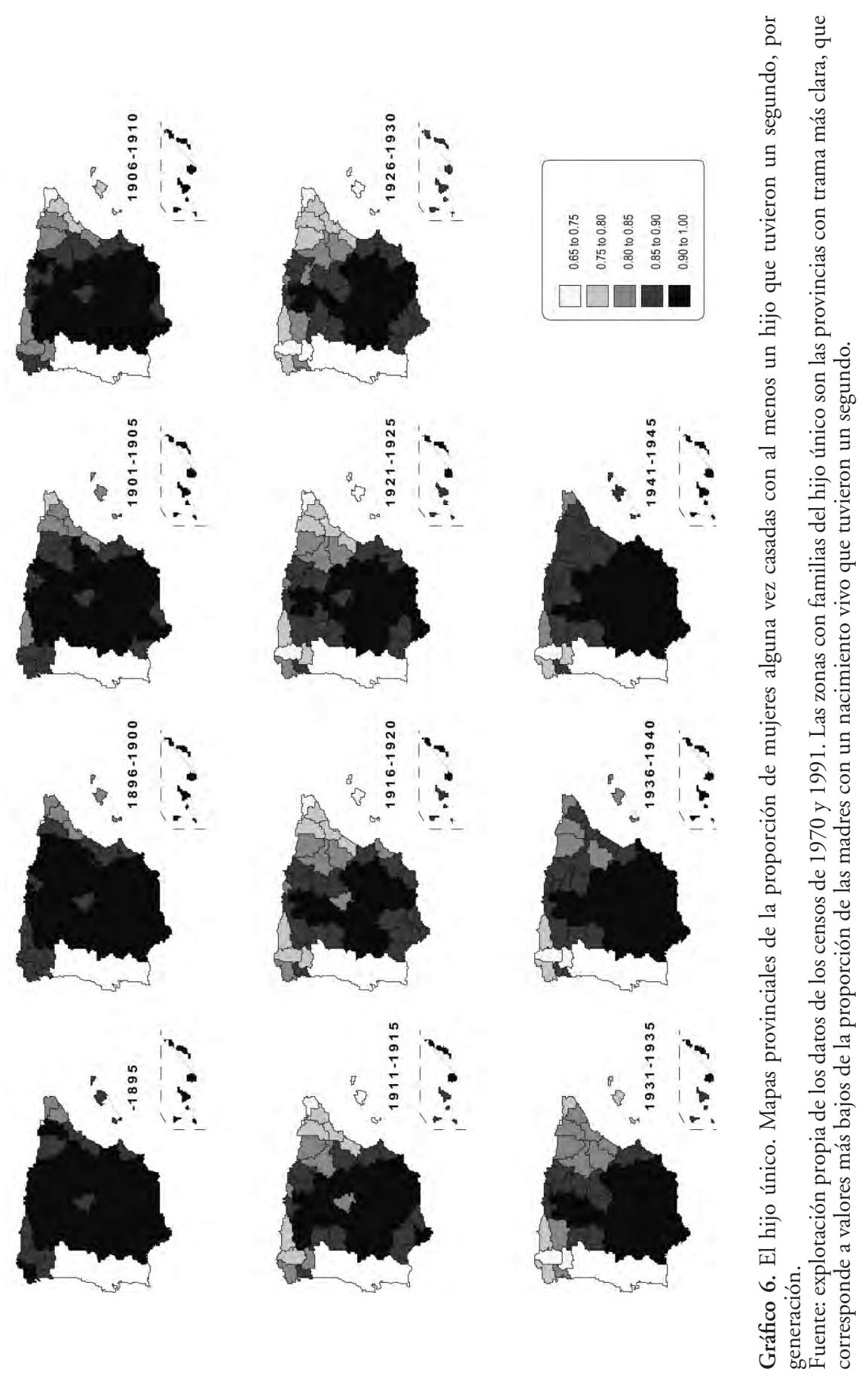
mujeres. Otra diferencia importante de este indicador y el anterior es que mide la fecundidad sin tener en cuenta la posible muerte anterior del hermano o de la hermana mayor, cuando el $a_{0}$ relaciona por fuerza los primeros nacimientos con efectivos de madres en vida. Esta última observación es importante, ya que la mortalidad infantil bajó de forma muy rápida durante todo el siglo XX. Por consiguiente, cada vez era menos necesario para las familias tener nacimientos de reemplazo, es decir segundos, terceros o nacimientos de orden superior que podían suplir la muerte de un hermano o de una hermana mayor.

En el caso de los nacimientos de segundo rango, vemos en los mapas que el descenso de la mortalidad hizo aflorar zonas en las que un amplio número de familias seguía una pauta de fecundidad que podemos llamar de hijo único. Si nos centramos, por ejemplo, en los datos de las generaciones nacidas durante la década de 1920, observamos la existencia de una zona mediterránea con una baja proporción de segundos nacimientos entre las mujeres con un hijo y, por lo tanto, una elevada proporción de madres con un solo hijo. En esta zona, destacan, con los niveles más bajos de segundos nacimientos, las provincias de Gerona y Baleares. Esto no nos sorprende, pues concuerda con la bien conocida tradición catalana del hereu, conjuntos de reglas y leyes que favorecen la herencia desigualitaria con el objeto de evitar la fragmentación de las propiedades. Este propósito y esta tradición conducen lógicamente a la adopción de una determinada estrategia de fecundidad para tener un solo descendiente a la edad de transmisión de la herencia, y así evitar los conflictos o las situaciones de injusticia con los hermanos desfavorecidos. Es más sorprendente observar que en al menos dos provincias de Galicia, Lugo y Orense, un amplio grupo de familias seguía un modelo de fecundidad parecido, sin que hayan trascendido hasta ahora noticias acerca de una tradición del herdeiro. Es también interesante notar que el aumento de la fecundidad a partir de la década de 1950, o de los años del baby boom, atenuó los efectos de este modelo sobre la fecundidad, con la excepción de estas dos provincias gallegas, donde el bajo nivel de $a_{1}$ se mantuvo hasta la generación más joven observada.

Otro aspecto que conviene resaltar, en relación con la perspectiva de este trabajo, es que la baja fecundidad para los hijos de segundo rango en zonas de Galicia y del Mediterráneo se explicaba probablemente por situaciones económicas o tradiciones que no podemos calificar de «modernas», puesto que corresponden a la voluntad de las familias de transmitir las propiedades de generación a generación, sin hacer peligrar la continuidad de pequeñas empresas agrícolas, artesanales o comerciales, lo que significaría seguir así normas seculares. Sin embargo, las familias que seguían el modelo del hijo único utilizaron métodos contraceptivos después del primer nacimiento, cuando el descenso de la mortalidad infantil hizo desaparecer la necesidad de segundos o terceros nacimientos de reemplazo. En definitiva, estos comportamientos de fecundidad «modernos», que seguramente entraban en conflicto con ideas religiosas, respondían a normas ligadas a situaciones sociales y económicas que pertenecían a la sociedad tradicional y que iban desapareciendo con la industrialización y la urbanización. 


\section{La progresión de la limitación de la fecundidad}

Como hemos visto anteriormente, en el gráfico 1, el proceso de limitación de la fecundidad en España afectó principalmente a los rangos superiores, a partir del tercer hijo. El conjunto de mapas del gráfico 7 visualiza las diferencias a nivel provincial en la cronología de la adopción de comportamientos de limitación de la descendencia. De forma general, este proceso se adelantó en las zonas del este de la Península, a partir de la provincias catalanas, fue ganando poco a poco las provincias mediterráneas más al sur y luego zonas del centroeste y del noroeste. El resultado para las generaciones más jóvenes examinadas es una partición en tres grandes zonas homogéneas: una con un mayor nivel de la fecundidad de rango 3 en las provincias del sur, otra de menor nivel en el norte, separadas por un grupo de provincias en el centro de España y en torno al País Vasco con un nivel intermedio. Esta secuencia de mapas invita a realizar una interpretación en términos de difusión de comportamientos, ya que, visualmente, tenemos la impresión de que se sigue un proceso que empieza en Cataluña y llega al resto de España hacia el sur y el centro. Pero tenemos que matizar esta impresión, a partir de la observación de la presencia de un segundo foco de reducción de la fecundidad, centrado de nuevo, como en los mapas de $a_{1}$, sobre Galicia y Asturias, que no tiene, al inicio del proceso, puntos de contacto con el primer foco centrado sobre Cataluña.

Otra matización importante es que, como hemos visto, el control de la fecundidad no se limitó a los terceros y más nacimientos, sino que afectó de forma significativa también a los segundos nacimientos en poblaciones rurales de Galicia, como en Lugo y Orense, y del Mediterráneo, como en Gerona y Baleares. Esta limitación de la fecundidad después del nacimiento del primer hijo no se extendió a otras zonas, por lo cual tenemos que diferenciar claramente entre el comportamiento "moderno", de uso de contracepción, y las necesidades de las familias, que en algunas provincias se traducían en la reducción de la fecundidad a partir del tercer o el cuarto hijo, y en otras por reducirla desde el segundo. En términos generales, podríamos plantear la discusión como una confrontación entre, por una parte, la oferta de pautas de comportamiento y de métodos de control de la fecundidad y, por otra, la demanda por parte de las familias de mecanismos para ajustar su tamaño y su composición a situaciones sociales y económicas cambiantes. El debate sobre la transición demográfica y, en concreto, sobre las causas del descenso histórico de la fecundidad, a menudo se ha planteado de forma exclusiva en términos de la oferta de métodos y hábitos, mucho más que en función de la demanda o las necesidades de las familias. Así, se ha considerado que las diferencias a nivel territorial en la cronología del descenso de la fecundidad se podrían explicar esencialmente en términos de difusión, es decir que los comportamientos modernos y las técnicas novedosas se difunden territorialmente desde las zonas avanzadas hacia las más atrasadas. Esta argumentación se defiende, por ejemplo, con mucha fuerza en un artículo de Cleland y Wilson (1987). Estos autores, basándose en gran parte en los resultados del proyecto de Princeton, sostienen que 

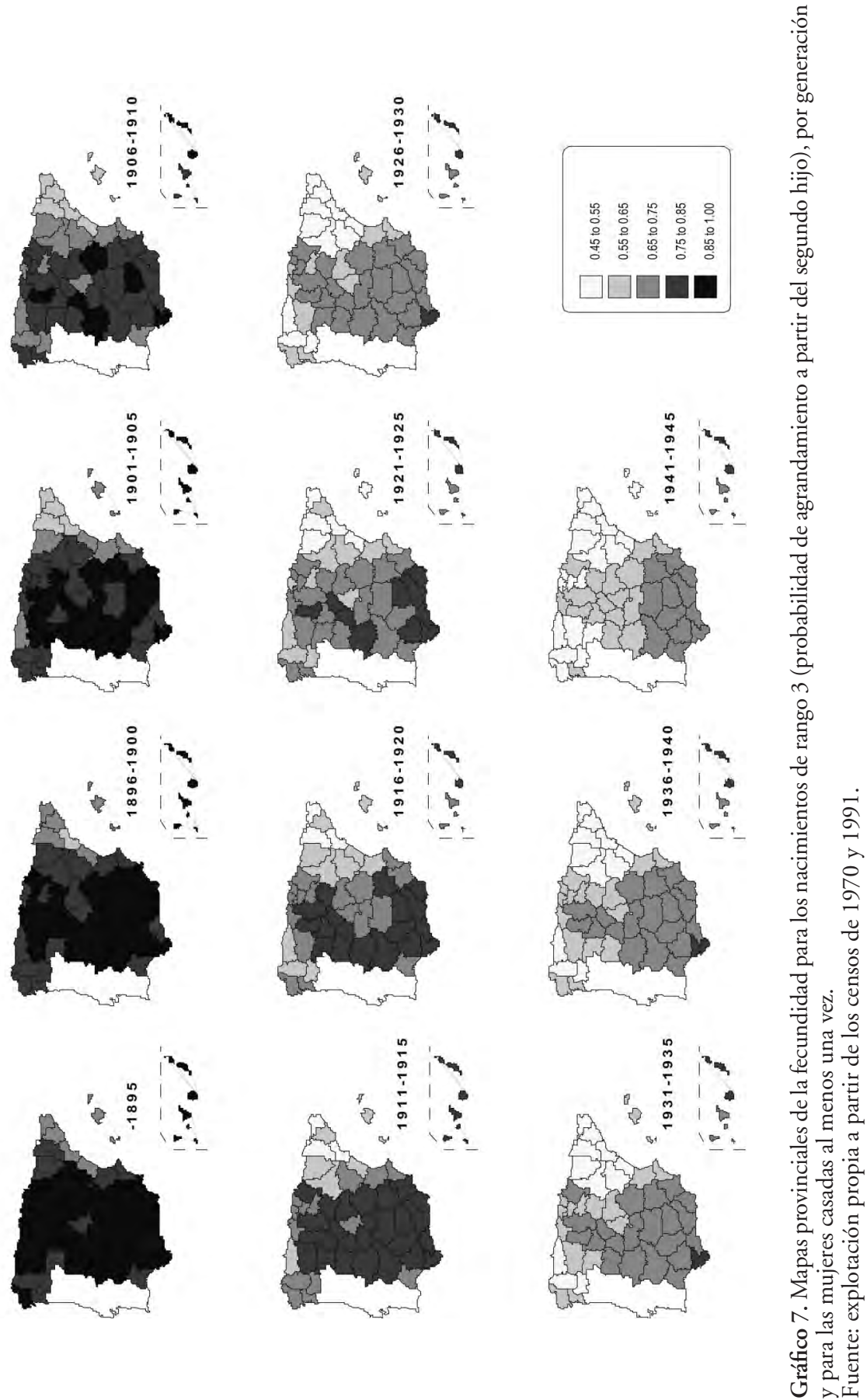
las teorías económicas o antropológicas que explican la fecundidad, su nivel y evolución, por la demanda de hijos, no sirven para explicar la transición de la fecundidad en Europa. Estos autores remiten, para explicar el descenso histórico de la fecundidad, a factores ligados a la oferta, como la difusión de métodos y de comportamientos novedosos socialmente asumibles, porque son practicados por un grupo adelantado de familias. A nuestro entender, las diferencias territoriales en la evolución de la fecundidad por rango en España contradicen esta explicación basada en exclusiva en la oferta de técnicas y comportamientos novedosos. El control de la fecundidad se extendió rápidamente en zonas con niveles de desarrollo social y económico muy diferentes, como es el caso de las provincias de Cataluña y Galicia, como consecuencia de la existencia de modelos familiares y de sistemas de herencia tradicionales. Es decir que el cambio en las necesidades de las familias, en respuesta al descenso rápido de la mortalidad infantil, desempeñó un papel central en la evolución de los niveles de fecundidad y la extensión de su control, mucho más que la oferta de los nuevos métodos y comportamientos.

Este debate se puede plantear también a partir de una reflexión sobre los indicadores del control de la fecundidad. Así, el uso de una única probabilidad de agrandamiento como indicador de control, por ejemplo la $a_{2}$, tiene claras limitaciones, ya que su nivel puede variar de una provincia a otra sin que la explicación sea la adopción de métodos de contracepción, sino, por ejemplo, por las diferencias en la duración del periodo de lactancia, que afecta de forma directa al intervalo entre nacimientos y, como consecuencia, puede reducir la fecundidad. Otra observación es que el control se puede producir en una provincia a partir de un rango, por ejemplo del segundo, pero afectar más a partir del tercer hijo en otra. Por lo cual, para estudiar los efectos de la adopción de los comportamientos de reducción de la descendencia, es mejor tener en cuenta el nivel de la fecundidad para todos los rangos a la vez. Presentamos, en la sección siguiente, un método de detección del control que va en esta dirección. Los resultados de la aplicación de este método constituirán una nueva base para poder avanzar en el debate de las causas del descenso histórico de la fecundidad.

\section{Estudio del control de la fecundidad a partir de la generación 1896-1900}

La comparación de las curvas de las probabilidades de agrandamiento de las cuatro provincias presentadas en el gráfico 8 ilustra de forma ejemplar las diferencias a nivel territorial en el progreso del control de la fecundidad. Las dos primeras, Álava y Granada, presentan un perfil de convexidad de las curvas en la primera generación observada, de 1896 a 1900, que es indicativo de la ausencia de control de la fecundidad, cuando, para esta misma generación, observamos que el perfil en las provincias de Huelva y sobre todo de Tarragona tiene la concavidad característica de la presencia de control. En las dos primeras provincias, las generaciones nacidas entre principios de siglo y 1941-1945 recorrieron un camino considerable en la adopción de métodos de control. Las 

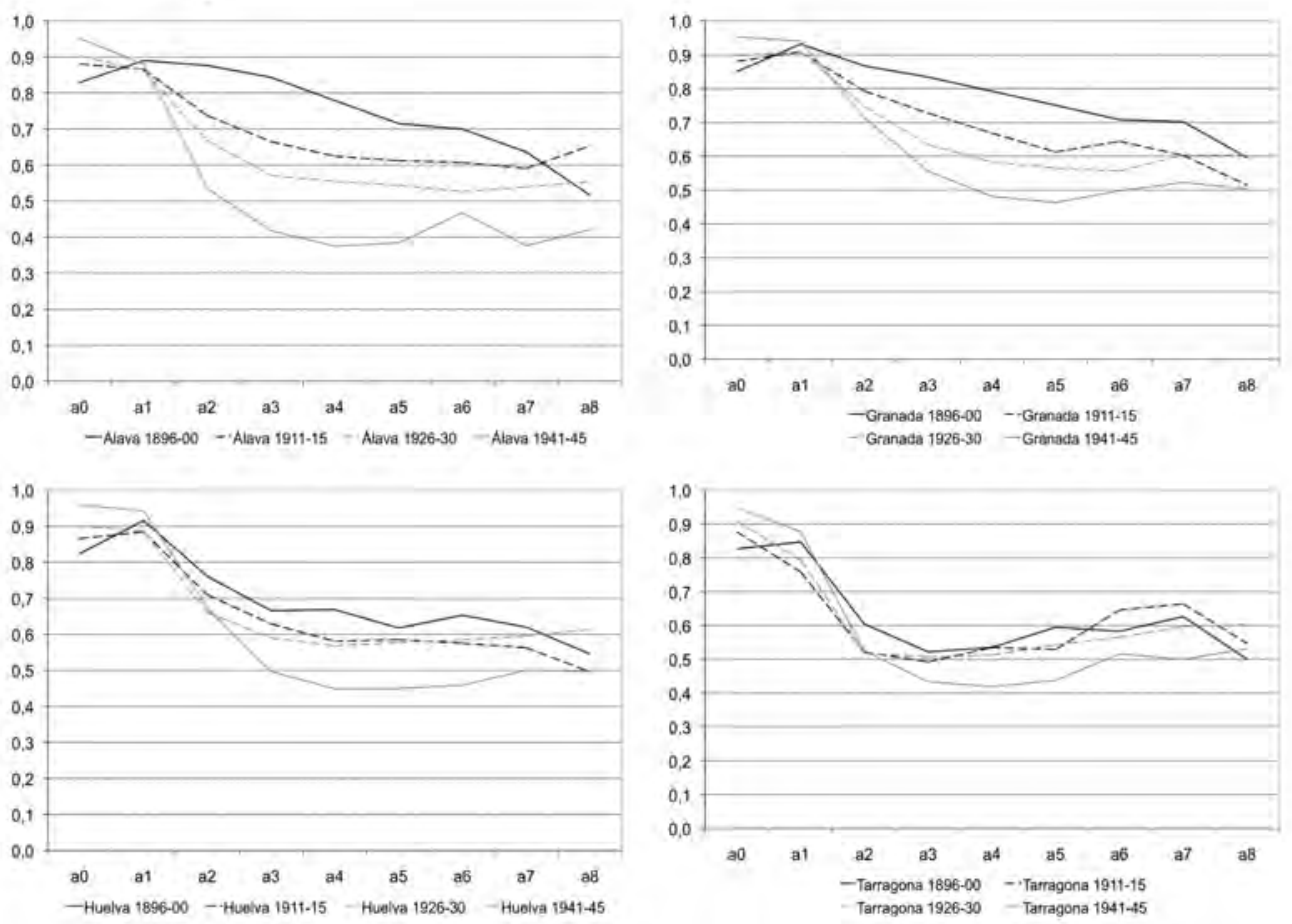

Gráfico 8. Progresión del control de la fecundidad. Probabilidades de agrandamiento de la generación 1896-1900 a la generación 1941-1945 para cuatro provincias seleccionadas.

Fuente: explotación propia de los datos de los censos de 1970 y 1991.

probabilidades de los rangos más afectados, del tercer, cuarto y quinto nacimiento, descendieron entre un 25 y un $50 \%$ del nivel correspondiente a la generación más antigua.

Se puede ir más lejos en el análisis de estas curvas y, en este caso, observar que, en poblaciones que no controlan su fecundidad, como el Québec del siglo XIX, el logit de las probabilidades a partir de la segunda $\left(a_{1}\right)$ se alinean sobre una recta, cuando, en cambio, en poblaciones donde el control es muy extendido, tenemos una curva con un punto de inflexión en torno al tercer hijo (gráfico 9). Si el logit de las probabilidades no se alinea sobre una recta, esto se debe a un control de la fecundidad, como lo muestra bien Sanderson (2000). Para poder estimar el grado de la linealidad, calculamos, para cada provincia, una regresión del valor de estos logits en relación con el rango de nacimiento a partir del segundo nacimiento, es decir, del valor de $a_{1}$. Si el valor del coeficiente de correlación al cuadrado (coeficiente de determinación) del ajuste es próximo a uno, entonces podemos considerar que la población estudiada no 


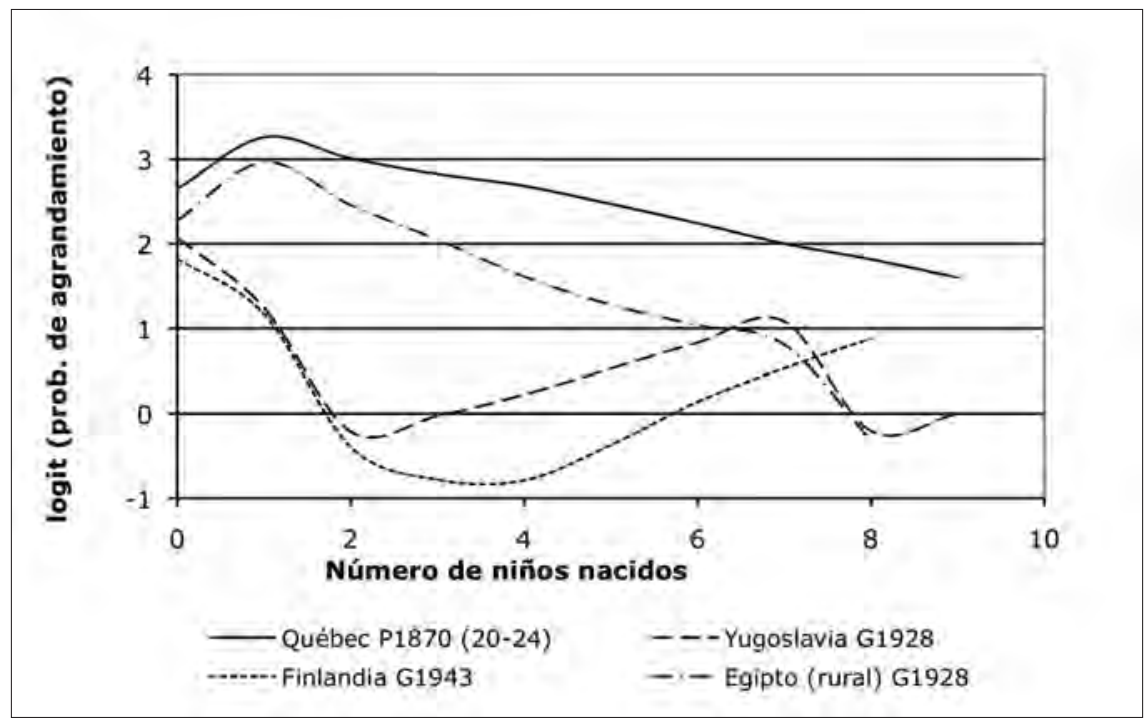

Gráfico 9. Criterio gráfico de detección del control de la fecundidad a partir de las probabilidades de crecimiento. Ejemplo de poblaciones con y sin control de la fecundidad. Nota: los datos representados son el logit de la probabilidades. Para Québec, datos de mujeres no solteras, casadas a los 20-24 años antes del año 1876 (Henry, 1953); antigua Yugoslavia, mujeres entre 60-64 años en el censo de 1991; Finlandia, mujeres de 45-49 años en el censo de 1991, y Egipto, mujeres casadas al menos una vez, de 45-49 años de edad en el censo de 1976, que vivían en zonas rurales (United Nations, 2000). Los valores de los coeficientes de determinación de las rectas a partir del primer nacimiento $\left(a_{1}\right)$ son los siguientes: Québec: 0,997; Egipto: 0,960; Finlandia: 0,050, y Yugoslavia: 0,001. Se puede considerar, pues, que las mujeres de Québec y de Egipto no controlaban su fecundidad, y que la proporción de las mujeres yugoslavas y finlandesas que la controlaban era muy elevada.

practicaba el control de la fecundidad. Para ser más exactos, no practicaba un control de tipo stopping, es decir, en función del número de hijos ya nacidos, con el propósito de limitar el tamaño de la familia a una cantidad preconcebida. $\mathrm{Al}$ revés, para las provincias con valores más bajos de este coeficiente, el alcance del control será importante. Pero no podemos ir más lejos que hablar en términos o bien de ausencia de control, o bien de control significativo, ya que todavía no se ha encontrado una fórmula para convertir los valores de este coeficiente en proporciones de mujeres que utilizaron el control de la fecundidad a lo largo de su vida fértil. Así, no se puede afirmar que un valor más bajo del coeficiente de determinación en una provincia que en otra signifique forzosamente que haya una proporción más elevada de mujeres que controlen la fecundidad.

Hemos aplicado este método a las series provinciales de las probabilidades de agrandamiento y, concretamente, a las de la generación más antigua sobre la cual disponemos de datos, es decir, las mujeres nacidas en el periodo 1896-1900. 


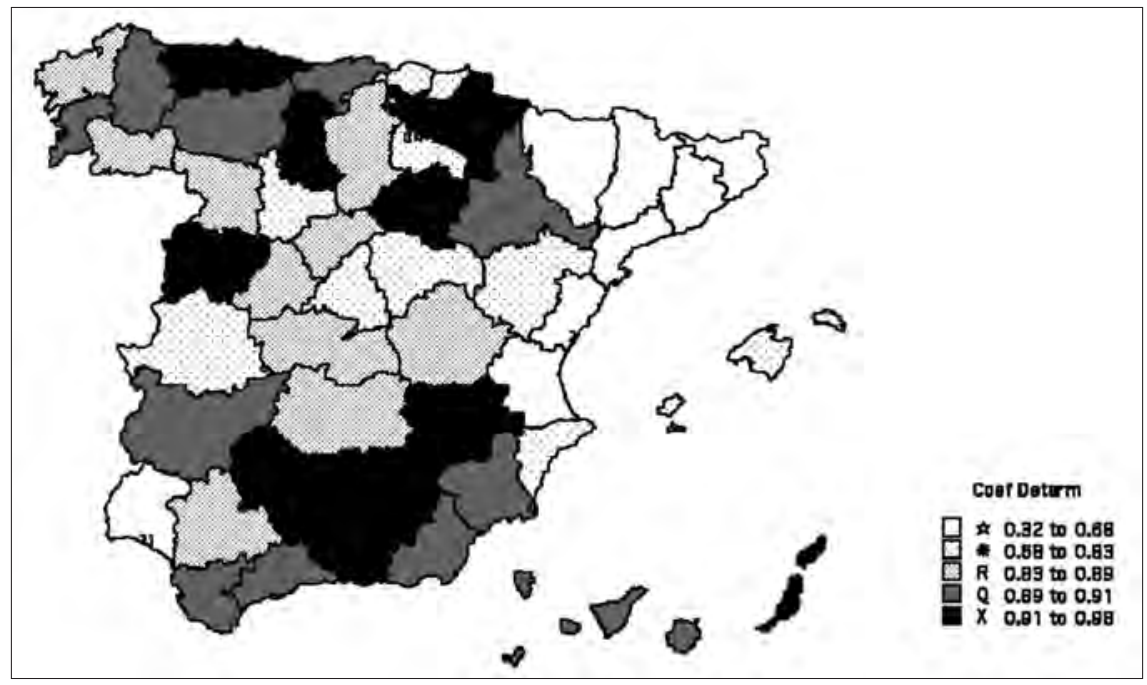

Mapa 1. Grado de control de la fecundidad en la generación 1896-1900, por provincias. Valores del coeficiente de determinación de la regresión del logit de las probabilidades de agrandamiento en función de los rangos de nacimiento.

Nota: cálculo del coeficiente de determinación provincial $\left(R^{2}\right)$ a partir del logit de las probabilidades de agrandamiento de la segunda $\left(a_{1}\right)$ hasta la novena $\left(a_{8}\right)$ en función del rango de nacimiento, para las generaciones nacidas en el periodo 1896-1900. Los datos son los mismos que en el gráfico 8 , pero para todas las provincias.

Los resultados se muestran en el mapa 1 y son remarcables al menos por dos razones. En primer lugar, porque este mapa difiere del que mostraba los valores de $a_{2}$ para la misma generación (gráfico 7). Así, hay provincias con bajo nivel de $a_{2}$ y control importante de la fecundidad (en Cataluña), zonas con alto nivel de $a_{2}$ y sin control de la fecundidad (en Andalucía), pero también, y es una sorpresa, muchas provincias con alto nivel de $a_{2}$ y un control importante (provincias de las dos Castillas y de Extremadura). En cambio, es tranquilizador ver que no hay provincias con bajo nivel de $a_{2}$ sin indicios de control, puesto que, en caso contrario, podríamos dudar de la efectividad del método de detección. Esto confirma la idea de que un alto nivel de la fecundidad legítima, total o de un rango determinado, no es una prueba suficiente de la ausencia de control.

En segundo lugar, este mapa nos indicaría que el control de la fecundidad era bastante más generalizado y extendido a lo largo de la geografía española de lo que nos podrían hacer pensar los mapas anteriores, sobre todo los referidos a los valores de $a_{1}$ y $a_{2}$. Así, no sorprende el hecho de que la población de provincias del Mediterráneo usase la contracepción de forma importante para nacimientos que se produjeron a partir de la década de 1910, pero sí sorprende que poblaciones de provincias muy alejadas geográficamente de las zonas 
adelantadas del Mediterráneo, como Huelva, y otras provincias del interior, como Valladolid, Cáceres, Guadalajara y La Rioja, tuviesen una configuración de la fecundidad por rango parecida a la catalana para la misma generación. $\mathrm{Y}$, a la inversa, es de nuevo una sorpresa constatar que en provincias tan próximas a núcleos de industrialización como Navarra y Álava, las mujeres nacidas a finales del siglo XIX que tuvieron a sus hijos en la década de 1920, no usasen métodos de control de la fecundidad y, en este aspecto, se pareciesen a las mujeres de las provincias agrarias del interior de Andalucía.

Pero lo que nos muestra este mapa es que no debemos considerar el control de la fecundidad como un comportamiento novedoso, o hablar de los métodos anticonceptivos como de técnicas desconocidas, y decir que los cambios analizados son consecuencia de un proceso de difusión de la información, que se hubiera tenido que producir antes de que las familias pudiesen hacer uso de los métodos modernos. Esto es claramente contrario a la realidad, puesto que la mayoría de las familias españolas conocían estos métodos, y muchas de ellas ya los utilizaban antes del descenso rápido de la fecundidad de la primera mitad del siglo XX. Entonces, destacar como factor de cambio la oferta de modelos de comportamiento o de técnicas nuevas tiene poco sentido. Las familias que no controlaban su fecundidad conocían las técnicas tradicionales de contracepción, pero no tenían necesidad de usarlas, porque realmente necesitaban tener hijos para compensar la alta mortalidad, para que las ayudasen, para satisfacer algunas de sus necesidades y para que cuidasen de ellas.

\section{Conclusiones}

El propósito principal del proyecto de Princeton era conocer los factores determinantes del inicio del declive de la fecundidad. Se consideraba este descenso como una ruptura entre un periodo previo en el que las familias no limitaban su descendencia a un periodo en el que se difunden los métodos contraceptivos y aumenta de forma rápida la proporción de mujeres que hacen uso de ellos. El estudio de la transición de fecundidad de las provincias españolas, a partir de un análisis por rango, nos conduce a otro planteamiento: el descenso histórico de la fecundidad no se explica por la introducción de técnicas o comportamientos novedosos, ya que, aunque muchas familias no limitaban su descendencia, conocían los métodos para hacerlo, y la prueba es que una parte significativa de las familias ya los usaban.

El mensaje de este trabajo es que el análisis de la evolución de la fecundidad no debe limitarse al uso de indicadores agregados que miden el valor total de la fecundidad, sino que, al contrario, la formación de la descendencia se decidía paso a paso, hijo tras hijo, y es de esta forma como debe analizarse. La evolución global de la fecundidad puede esconder ritmos contrapuestos, por ejemplo en el caso de España y en el de muchos otros países europeos, donde, durante la transición, aumentó la fecundidad de los primeros y los segundos hijos, cuando de forma simultánea disminuía la fecundidad de rango 3 y más. Como el aumento de las primeras probabilidades o el descenso de las últimas 
no se produjo de forma igual en todas las provincias, analizar la cronología de la transición a partir de la evolución del total de la fecundidad total es arriesgado y, además, puede llevar a conclusiones erróneas.

\section{Referencias bibliográficas}

CABRÉ, A. (1999). El sistema català de reproducció. Barcelona. Proa. La Mirada Social.

Cleland, J. y Wilson, C. (1987). «Demand theories of the fertility transition: an iconoclastic view». Population Studies, 41 (1), 5-30.

COALE, A. y TRusSeL, J. (1974). «Model fertility schedules: variations in the age structure of childbearing in human populations». Population Index, 40 (2), 185-258.

Coale, A.J. y WatKins, S.C. (1986). The decline of fertility in Europe. Princeton. Princeton University Press.

DAVID, P.A. y SANDERSON, W.C. (1990). "Cohort parity analysis and fertility transition dynamics: Reconstructing historical trends in fertility control from a single census». Population Studies, 44 (3), 421-445.

Devolder, D. y Merino, M. (2004). La infecundidad y fecundidad de las familias desde una perspectiva longitudinal en los paises occidentales. VII Congreso de la Asociación de Demografía Histórica. Granada.

Devolder, D.; Nicolau, R. y Panareda, E. (2004). La fecundidad de las generaciones españolas nacidas en la primera mitad del siglo XX: Un estudio a nivel provincial. VII Congreso de la Asociación de Demografía Histórica. Granada.

Dyson, T. y Murphy, M. (1985). «The onset of fertility transition». Population and Development Review, 11 (3), 399-440.

Gil Alonso, F. (1997). «Las diferenciales territoriales en el descenso de la fecundidad en España: Aproximación a su estudio a partir de datos censales sobre fecundidad retrospectiva». Boletín de la ADEH, XV (II), 13-54.

Henry, L. (1953). Fécondité des mariages: Nouvelle méthode de mesure. París. INED. Presses Universitaires de France.

MORGAN, S.P. (1991). «Late nineteenth and early twentieth-century childlessness». American Journal of Sociology, 97 (3), 779-807.

OKUN, B.S. (1994). «Evaluating methods for detecting fertility control: Coale and Trussel's model and cohort parity analysis». Population Studies, 48 (2), 193-222.

ReCaño, J. y LuXAN, M. (1997). Un estudi de la fecunditat de Catalunya a partir de les dades del cens de 1991. Bellaterra. Centre d'Estudis Demogràfics.

RowLAND, D.T. (1998). Cross-national trends in childlessness, research school of social sciences. The Australian National University.

RYDER, N.B. (1986). «Observations on the history of cohort fertility in the United States». Population and Development Review, 4 (12), 617-643.

SAEZ, A. (1979). «La fécondité en Espagne depuis le début du siècle». Population, 6, 1007-1022.

SANDERSON, W.C. (2000). A users' guide to the joys and pitfalls of cohort parity analysis. Stanford. [http://siepr.stanford.edu/conferences/Sanderson.pdf]

United Nations (2000). Demographic yearbook, historical supplement: 1948-1997. Nueva York.

Wrigley, E.A.; DAVIES, R.S.; Oeppen, J.E. y otros (1997). English population history from family reconstitution, 1580-1837. Cambridge. Cambridge University Press. 